\title{
Debates y redefiniciones del patrimonio cultural
}

\author{
Jorge Echavarría Carvajaj"
}

Recibido: 29 de febrero de 2016

Enviado a pares evaluadores: 10 de marzo de 2016

Aprobado por pares evaluadores: 31 de marzo de 2016

Aprobado por comité editorial: 14 de abril de 2016

\section{RESUMEN}

Este artículo analiza los cambios que la noción de patrimonio cultural ha estado experimentando en las últimas décadas, motivados por la tecnología, así como por redefiniciones teóricas, sociales y culturales en el contexto de la globalización y sus secuelas. El artículo sugiere que es necesario tomar en consideración los cambios para obtener un conjunto más preciso de herramientas políticas y conceptuales para la gestión del patrimonio, y para encontrar una conexión adecuada y renovada entre la gente y su cultura y patrimonio.

Palabras clave: Patrimonio cultural, cultura, gestión patrimonial, políticas culturales, tecnologías de la memoria, globalización cultural, cambios. 


\section{Debates and Redefinition of Cultural Legacy}

\section{ABSTRACT}

This articles analyzes the changes that

number of political and conceptual tools cultural legacy notion has experienced during the last decades, boosted by technology and theoretical, social, and cultural redefinitions within the globalization context and its consequences. The article suggests the necessity of taking changes into consideration in order to obtain a more accurate to manage legacy and find a suitable and renewed connection between people and their culture and legacy.

Key words: Cultural legacy; culture, patrimonial management; cultural policies; memory technologies; cultural globalization; changes. 


\section{A modo de preámbulo}

Como puede deducirse del título y el resumen, la modesta ambición de estas páginas se centra en señalar algunos de los debates que surgen dentro del cada vez más inabarcable campo del patrimonio cultural, decantándose por aquellos problemas que proponen nuevas direcciones y respuestas. En este sentido, se acogen a una serie de autores y teorías, vinculados unos directamente con la reflexión sobre el patrimonio, mientras otros, desde orillas teóricas muy diversas, aportan miradas que complementan e interrogan las cómodas posiciones asumidas usualmente desde los discursos y prácticas oficiales en torno a la herencia cultural, sus propósitos y modos de funcionamiento. Así como Borges pensaba lo que debía ser un curso de literatura, este artículo pretende ser una guía de lecturas anotadas que detonen potenciales reacciones y ulteriores diálogos, enfatizando la necesidad de nuevas formas de pensar y actuar en el campo patrimonial.

\section{Triunfo y crisis patrimonial}

El innegable triunfo y presencia central del patrimonio cultural en la cultura contemporánea es constatable en el creciente número de museos, de declaratorias de patrimonio en diversas escalas (cascos históricos, paisajes, costumbres y tradiciones...), y del auge de estudios, debates y publicaciones en el tema. Como derivado de este fenómeno, surgen innumerables preguntas acerca de los criterios de sostenibilidad, de apropiación y compromiso social, de la propiedad y responsabilidad, de su industrialización y contribución a la economía, de su conservación en medio del cambio, acerca de su regulación o liberación de trabas y legislaciones, en torno a su difusión con fines pedagógicos o su aprovechamiento turístico, del grado y pertinencia del discurso académico para describir las acciones reales de apropiación, de los límites mismos de lo patrimonializable y de lo museable y las decisiones políticas e ideológicas tras una declaratoria en tal sentido, etc. ${ }^{1}$. Muchos de estos interrogantes no son, por supuesto nuevos, pero muchos otros se cocinan al calor de las nuevas formas de habitar y asociarse que las tecnologías y las formas globalizadas de relación y contacto propician, reclamando respuestas acordes con sus perfiles, interpelando a la epistemología ${ }^{2}$, a las reflexiones sobre la cultura y la sociedad, a los discursos y praxis políticas, a la economía de los bienes y de los símbolos, entre otros campos.

\footnotetext{
1 Ya en 1999, un año cercano cronológicamente pero alejado inimaginablemente por obra y gracia de la generalización de las tecnologías y sus impactos socioculturales, François Matarasso y Charles Landry (1999) identificaron 21 dilemas estratégicos para las políticas públicas en cultura, muchos de ellos con implicaciones y derivas patrimoniales complejas y aún hoy sin solución. En adelante, cualquier cita directa o indirecta en un idioma diferente al español es de mi responsabilidad.

$2 \quad$ Particularmente interesante es Staiff, Russell, Bushell, Robyn and Watson, Steve (eds.) 2014.
} 
Extrema occursum, los extremos se tocan: el apabullante éxito del patrimonio viene acompañado por innumerables quejas, debates y reclamos acerca de su validez, aporte y responsabilidad. Sin embargo, muchas de estas encrucijadas siguen partiendo, rutinariamente y sin examen previo, de un conjunto de puntos fijos e incuestionables, culpables, tal vez en mucho, de que las soluciones ni siquiera se contemplen o que se dilaten indefinidamente, al plantearse, a partir de premisas, que ya no dan cuenta de los fenómenos y conceptos que realmente permitirían dar otras orientaciones al patrimonio y a su cambiante papel en nuestras culturas ${ }^{3}$.

Es claro, eso sí, que el nacimiento de la noción de "patrimonio cultural" es consustancial al proyecto político moderno, y nace como antídoto contra el vandalismo revolucionario, iniciado en 1792 con la demolición de la estatua de Luis XIV, erigida en la plaza Vendôme ${ }^{4}$. La iconoclastia contra las tumbas reales en Saint Denis, en el año siguiente ${ }^{5}$, y el desatado proceso allí incubado contra todo rastro de memoria de la monarquía lleva a la Convención a proteger tales monumentos y memorias, recogiendo discusiones previas sobre el llamado, recién entonces, "patrimonio nacional"6. Así, se nacionaliza un conjunto de bienes bajo la etiqueta de patrimonio, extendiendo a los productos culturales un concepto de origen económico, se crea el monopolio de una memoria social exclusivamente centrada en lo patrimonial, y se da partida a un proceso político, social y cultural que es hoy tan conspicuo y extendido.

Sin embargo, si el proyecto moderno que vio nacer este concepto y sus grandes relatos está en este momento, al menos, bajo revisión, pareciera que la sacralización laica patrimonial siguiera incólume y sin vacilaciones, a pesar de que sus usos políticos, identitarios y pedagógicos ya no se ajustan plenamente con dicho proyecto moderno y sus mutaciones contemporáneas. Las fronteras nacionales son hoy porosas a fuerzas y vectores (tecnologías, migraciones, indus-

3 Rizando el rizo, como gráficamente se dice en una simpática locución peninsular, cada aspecto del dispositivo patrimonial está sujeto hoy a debates particulares a su propia área de competencia. Por ejemplo, en lo que respecta a la preservación, ver el texto de Richmondy Bracker, Alison (eds.), 2009. Este texto vuelve en coordenadas actuales a la revisión de asuntos como la conservación y sus dilemas éticos, la restauración, la magnitud de las intervenciones, etc. Para lo relativo a la dimensión educativa en la agenda patrimonial es particularmente útil (Atkinson, 2014).

Al lado de ello, los estudios sobre los impactos que tienen las dinámicas sociales en el patrimonio son también abundantes, por ejemplo Innocenti, P. (ed.), 2014.

4 Ver el muy documentado libro de Gamboni (2014).

5 Alexander Dumas, padre, tiene un detallado cuento al respecto: "Las tumbas de Saint Denis", disponible en: http://www.ciudadseva.com/textos/cuentos/fran/dumas/las_tumbas_de saint_denis.htm

6 "Fue François Puthod de Maisonrouge, un intelectual francés de segunda categoría, originario de Mâcon, quien habló por primera vez de patrimonio nacional en un discurso en la Asamblea Nacional pronunciado el 4 de octubre de 1790, para referirse a los monumentos. La fórmula tuvo muchísimo éxito entre los diputados franceses, que la adoptaron inmediatamente", como nos relata Avilés Flórez (2012), 78. El artículo, sin embargo, se apega al modelo UNESCO como si fuera el cierre absoluto de la definición patrimonial. 
trias culturales) hace unas décadas insospechados, haciendo que lo vernáculo y lo foráneo ya no sean oposiciones binarias simples, sino grados y mezclas de una variedad proliferante, en las que lo patrimonial se debate entre tensiones.

\section{Del origen a los cambios}

Si bien "la historia de todas las sociedades puede ser caracterizada como una lucha contra el olvido" (como nos recuerda Rusu, 2015, p. 384), las tecnologías informáticas y de gestión de información han llevado esta posibilidad de no olvido, antes balanceada en su contrapeso con lo recordable, a su exacerbación máxima. La información, poderoso activo económico, engulle cantidades ingentes de datos que se guardan y usan, proporcionando poder e ingresos a quienes los poseen. Este mismo programa se eleva a la categoría política de "derecho a la memoria", consagrando el "paradigma anamnésico de la existencia cultural" (Rusu, 2015, pp. 387), esto es, el guion de una cultura empeñada en no olvidar nada. Tal terror al olvido subyace las operaciones de patrimonialización cultural, y elude el que, contra este exceso de memoria tecnológicamente mediado, el olvido es saludable. Ya Umberto Eco advertía que el gran inventario de información de Internet no constituía en sí mismo la memoria ${ }^{7}$ y, medio en broma, que habría de establecerse una especie de "arte del olvido", proyecto que concretaría Wienreich ${ }^{8}$.

Memoria como exceso y olvido como fármaco serían, en últimas, dos extremos a los que patrimonio cultural hoy debería mirar atentamente para definir su lugar.

Cuando aparece la noción de "patrimonio cultural" a la que subyacen las ya adoptadas oficialmente y concurrentes de "cultura" o de "políticas culturales", ni la tecnología de la información, ni el turismo masivo, ni las industrias culturales, ni la cultura globalizada eran las fuerzas jalonadoras tanto de la vida cotidiana como de las dinámicas patrimoniales ${ }^{9}$. El "afrancesamiento" de la UNESCO ${ }^{10}$ es un elemento ideológico crucial en estas definiciones que han seguido tras bambalinas de las políticas y prácticas patrimoniales: patrimonialización esencialmente como museificación ${ }^{11}$, énfasis en lo monumental, patrimonio como sinónimo de identidad, estetización como reespiritualización del referente patrimonial, acento en lo pedagógico, extensión de la idea de patrimonio a muchos ámbitos ${ }^{12}$, patrimonio como dispositivo preferente de la

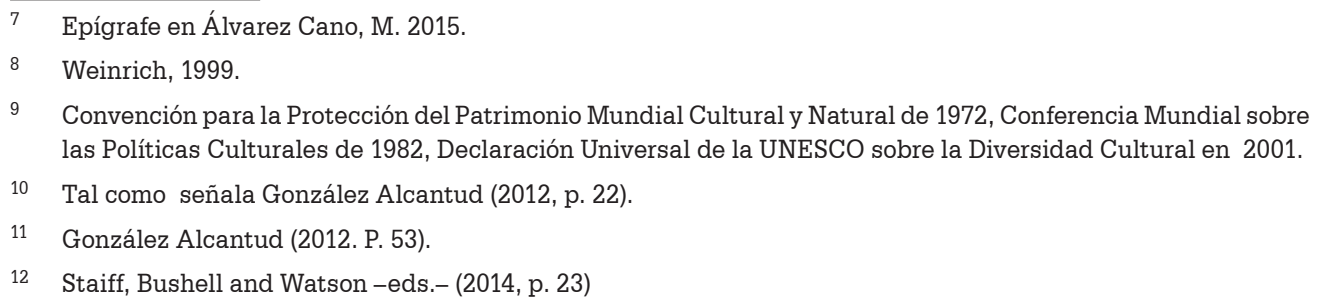

9 Convención para la Protección del Patrimonio Mundial Cultural y Natural de 1972, Conferencia Mundial sobre las Políticas Culturales de 1982, Declaración Universal de la UNESCO sobre la Diversidad Cultural en 2001.

10 Tal como señala González Alcantud (2012, p. 22).

11 González Alcantud (2012. P. 53).

12 Staiff, Bushell and Watson -eds.- (2014, p. 23) 
memoria social, lo patrimonial como lo opuesto a lo funcional y utilitario, lo visual como construcción y experiencia básica de lo patrimonial ${ }^{13}$, traslado mecánico de prácticas y conceptos patrimoniales occidentales a culturas muy diversas, etc. ${ }^{14}$.

Al tiempo, debe recordarse que los elementos culturales patrimoniales tomados como característicos de un grupo humano se usan como señales de etnicidad, estableciendo distinciones entre los diversos colectivos humanos, marcadores estos que crean, conservan y expresan tales singularidades, propiedad exclusiva del pueblo que las posee. Evidentemente, un sondeo histórico-arqueológico permite ver los préstamos, apropiaciones, reinterpretaciones y creaciones tras tales señales, además de evidenciar procesos contemporáneos de manipulación para obtener réditos turísticos o comerciales (exotismos, nostalgias, etc.), entre otros muchos. Como resalta Thouen (en Kasten-ed.-, 2014, p. 91), "la idea de que la cultura debe ser tratada como propiedad es principalmente derivada de una concepción relativista y romántica de la cultura, en tanto entidades mutuamente ligadas y que han de ser entendidas solo en sus propios términos". Este enfoque se muestra en conflicto abierto, y no resuelto, con las pretensiones de las nociones universalistas de cultura, de cuño ilustrado, y donde los rasgos de etnicidad serían meros "accidentes" que no empañan ni comprometen el destino común de la humanidad y "una visión del saber secular al servicio de los derechos del hombre y del progreso humano" (Wilson, 1998). Frente a esta sobrevaloración ilustrada de la razón y su cosmopolitismo, el romanticismo exalta las singularidades y la historia como marcas irreductibles de cada pueblo ${ }^{15}$. El argumento patrimonial moderno ha de tratar de confrontar estas dos dimensiones, pero, para hacerlo, su discurso y prácticas rutinarias dejan ver muchas fisuras.

Los cambios en estas nociones, lo mismo que los contextos y las dinámicas relacionales e impulsoras, aparecen hoy bien diferenciados: la extensión de lo patrimonial ha desbordado cualquier previsión. Los términos para describir esta obsesión patrimonialista no dejan duda del sesgo crítico en un amplio abanico de autores: "Delirio conmemorativo" u "obsesión memorialista", según Andrés Huyssen ${ }^{16}$; "memoria saturada", para Régine Robin ${ }^{17}$; "sobredosis identitaria", en Jean Claude Kaufmann" ${ }^{18}$; "autoarqueologización", según Charles S.

\footnotetext{
Staiff, Bushell and Watson -eds.- (2014, p. 84 ss).
}

Salazar, Juan Francisco y Bushell, Robyn in Staiff, Bushell and Watson -eds. (2014, pp. 187ss).

15 El filósofo y docente de la Universidad de Barcelona Gonçal Mayos (s. f.) propone que esta universalidad ilustrada, entendida como unidad, homogeneidad y continuidad, es un trasunto de la aplicación de un modelo de racionalidad geométrica, dominante en la primera modernidad, donde el afán de cuantificación, epítome de lo intelectual, rechaza cualquier singularidad no reductible a la medida.

16 Huyssen, A. 2002.

17 Robin, R. 2003.

18 Kaufmann, Jean-Claude, 2015. 
Maier ${ }^{19}$; acorde con González Alcantud, "furor patrimonialista" y "fetichismo patrimonial"20; "ilusión narcisista nacida del espejo patrimonial" en François Choay ${ }^{21}$; Lowenthal ve una "obsesión y posesión patrimonial" 22 , mientras que Ronström mira al patrimonio como una cruzada e imperativo moral hoy inexcusables $^{23}$.

Ante esta avalancha de adjetivos que ponen en duda el que tras este triunfo de la cultura patrimonialista haya una bondad innata y fuera de toda sospecha, se impone revisar la compleja trama de conceptos, discursos y prácticas que dan sustento a tal exceso ${ }^{24}$.

\section{Esencialismo y relativismo patrimonial}

Un punto de partida obvio para la fundamentación patrimonial es la memoria. Si la Modernidad prohijó la historia como el modelo para atrapar la memoria y convirtió a esta en su coto de caza exclusivo, nuestros tiempos parecieran haber dado un giro para hacer del patrimonio el paradigma que subsume historia, memoria, pasado colectivo, tradición, mito y cualquier otra manera de relación con el pasado. Esta nueva dirección hace que se esencialice el patrimonio, convirtiéndolo en un "imperativo categórico": el atentado y destrucción de los Budas de Bamiyán o cualquiera otro contra alguna obra que se considere como parte del patrimonio, suscitan una reacción de indignación moral que llama a acciones contundentes y de solidaridad internacional, incluso desproporcionadas en sus alcances y metas comparadas, por ejemplo, con otros ámbitos en donde está en juego la supervivencia como especie y donde no pareciera que las respuestas sean tan inmediatas y solidarias ${ }^{25}$. Otro efecto colateral a esta bulimia patrimonial ${ }^{26}$ y a su monopolio sobre la memoria resultaría en que una "obsesión con el patrimonio haya llevado a una detención de la creatividad contemporánea mientras presenta un sentido universal ficticio sobre la identidad humana"27. Es decir, el patrimonio da el visto bueno a ciertas identidades, narrativas y me-

\footnotetext{
19 Maier, C. 1988.

20 González Alcantud, J., 2012.

21 Choay, F. 2001.

22 Lowenthal, D., 1998.

23 Ronström, O., 2015.

24 Sin embargo, y en contravía de tales cambios, una somera revisión de los Planes de desarrollo cultural de los municipios (que se copian unos a otros y de administración en administración, en un ejercicio superficial para meramente cumplir con un requisito legal gubernamental), pone en evidencia que siempre, inexorablemente, se parte de las definiciones cultura, de patrimonio y de sus tipologías acuñadas hace más de 40 años y recogidas acríticamente.

25 Ni las crisis de refugiados, los desastres ambientales, la reducción del efecto invernadero, etc., para citar sólo algunas.

$26 \quad$ El adjetivo es también de González Alcantud (2012) p. 12.

27 Preocupación de Robert Hewison (1987) y Patrick Wright (1985) en Smith (2011, p. 59).
} 
morias, quitando a otras su franquicia de legitimidad, un expediente de cariz político que es esgrimida frecuentemente contra las minorías étnicas.

Frente a este esencialismo patrimonial, se ven aparecer voces discordantes: Bendix (2012), plantea que el patrimonio es creado, Bella Dicks (en Smith, 2011) apunta a su carácter de acto comunicativo ${ }^{28}$ y el que hoy hay modos de producir la "visitabilidad" (2004) ${ }^{29}$, mientras que la académica australiana Laurajane Smith (2011) nos recuerda que la persistencia de la noción objetual en lo patrimonial (en tanto se trata tanto de objetos materiales como de materializaciones de herencias inmateriales), que ha orientado los modos de investigar, legislar, difundir y proteger el patrimonio, nace cuando "el discurso patrimonial autorizado se desarrolló en Europa occidental en el siglo XIX, luego de la discusión de arqueólogos y arquitectos por la protección de la cultura material, que consideraban de valor innato y heredable", por lo que el discurso patrimonial se centra en objetos, paisajes, sitios, prácticas materializadas, etc., con un valor innato, bueno per se, universal y perenne. Smith propondrá, pues, frente a este sesgo y las obligaciones que conlleva (protección, transmisión inalterada al futuro, valor cultual, etc.), un nuevo modelo: "De hecho, para mí, el patrimonio no es la cosa, el sitio ni el lugar: el patrimonio son los procesos de creación de sentido y de representación que ocurren cuando se identifican, definen, manejan, exhiben y visitan los lugares o eventos patrimoniales"30, o "el patrimonio es algo activo, algo que se hace y no se posee" ${ }^{31}$, de modo que, reformulando y precisando lo planteado por el monumental trabajo de Pierre Nora $^{32}$, "los lugares se vuelven patrimonio debido a los actos de manejo, conservación y visitas que ocurren en ellos, y con ellos". ${ }^{33}$ Son evidentes los cambios de dirección que ello plantearía a las entidades encargadas de las políticas públicas sobre patrimonio, de un lado, y la irrelevancia de ciertas discusiones, por ejemplo las referidas a las farragosas delimitaciones entre patrimonios materiales e inmateriales y su tratamiento diferencial en políticas y prácticas, lo mismo que a revelar la ingenua falacia tras los discursos patrimoniales oficiales que migran de las distinciones entre bienes muebles e inmuebles a las de paisajes y sitios patrimoniales, sin realmente enfrentar que no se trata tanto de evolucionar en un concepto patrimonial, sino de poner en cuestión las bases esencialistas e identitarias desde donde se parte.

\footnotetext{
28 Ambos también citados por en Smith, 2011, p. 59.

29 Es decir, la cultura entendida como "algo adonde ir", título irónico de la introducción al libro de Dicks (2004).

30 Smith, 2011, p. 45.

31 Smith, 2011, p. 59. Aquí surgen muchas polémicas acerca de la "propiedad" intelectual y legal del patrimonio.

32 Nora, P. (ed.), 2001. Para Nora, "lo que hace del lugar un lugar de memoria es tanto su condición de encrucijada donde se cortan diferentes caminos de la memoria como su capacidad para perdurar y ser incesantemente remodelado, reabordado y revisitado "(Allier Montaño, 2008. P. 167). De hecho, la historia misma es en Nora uno de tantos "lugares de la memoria", una operación intelectual y laica.
}

33 Smith, 2011, p. 59. 


\section{La cultura y la identidad a debate}

Si examinamos el concepto de cultura, definida está en la ya archiconocida conceptualización adoptada por UNESCO como "el conjunto de rasgos distintivos espirituales y materiales, intelectuales y afectivos que caracterizan a una sociedad o a un grupo social" , claramente se resalta la idea de singularidad identitaria de una nación o grupo, en contravía evidente con el título del documento en el cual se hace esta -Declaración Universal sobre la Diversidad Cultural-: ¿una declaración universal de la singularidad?, lo que de nuevo nos retrotrae a la ya anotada disputa entre el universalismo cosmopolita ilustrado y el romanticismo singularizante. A ello puede contraponerse, de un modo bien productivo, el concepto manejado por Bernard Stiegler (1998, p. 193) ${ }^{34}$, en tanto "la cultura no es otra cosa que la capacidad de heredar colectivamente la experiencia de nuestros ancestros" 35 . Desde esta perspectiva, los componentes experiencial y comunicativo son esenciales en el obrar de una memoria cultural transmitida, dejando de lado aspectos colaterales sobredimensionados en la idea patrimonial moderna.

Podría afirmarse que la primera etapa del apogeo patrimonial estuvo signada por la apuesta ilustrada: la cultura, patrimonio común, es reivindicada por la humanidad entera, que crea los grandes museos modernos ${ }^{36}$ en cada potencia colonial, y que han de albergar esta herencia común: expolios, pillaje, expropiación, compra, etc., se justifican para divulgar este tesoro heredado por todos y puesto a disposición en los grandes centros creados para exhibirlos, preservarlos y atesorarlos. Hoy, la tesis neorromántica resurge con fuerza: se exige devolver a la cultura expoliada su "propiedad" patrimonial. ${ }^{37}$

$\mathrm{Si}$, por contraste, adoptamos el carácter sociosemiótico de ambas dimensiones, patrimonio y cultura, estas dejan de ser colecciones de cosas, prácticas y materializaciones, para pasar a ser actividades de producción de sentido, confrontaciones y negociaciones de sujetos y colectivos reales frente a significados culturales y sociales, no ya para refrendar un pasado mítico e intocable, sino para dar sentido al presente existencial a través de esa actividad de resignificación e interpelación. Si algo hay de "universal" en ello, es precisamente este mecanismo de permanente producción de sentido. Así, también ello nos pone

34 En alineamiento con los desarrollados sobre la técnica y la memoria en Leroi-Gourhan y Régis Debray.

35 No muy lejano del modelo mediológico de Debray (2001), donde la cultura es definida por los dispositivos de transmisión, "entre esferas espaciotemporales distintas" (p. 16).

36 Para Déotte (2013), el museo es uno de los primeros "aparatos" modernos.

37 Los mármoles del Partenón, el tesoro Quimbaya, los restos del galeón San José, la devolución de las obras robadas por los nazis (con dos películas recientes a cuestas, "La dama dorada" - Woman in Gold- de Simon Curtis, 2015 y "Operación Monumento"- Monuments Men" de George Clooney, 2014), ilustran varios de estos reclamos: ver en otros apartes de este trabajo, las glosas sobre las nociones que critican la idea de patrimonio como propiedad. 
de presente que el discurso patrimonial oficial o académico es solo uno de los muchos posibles, y que no puede arrogarse el monopolio de esta dimensión. De otro lado, se pone en evidencia el carácter controlador, normativo, elitista y narcisista del tal discurso patrimonial, y el proceso de deslegitimación de cualquier debate acerca de sus alcances.

La pluralización de los procesos de memoria, el carácter negociado del patrimonio, su descosificación llevan a plantear otros escenarios, como el propuesto por Ronström (2005, p. 3): "(...) cualquier producción de memoria colectiva o de pasados colectivos debe ser entendida en relación con otras producciones de tal tipo, a lo que denomino producción de $(\operatorname{mindscapes})^{38}(\ldots)$ ", esto es, una entidad tanto psíquica como material. Un mindscape nace de un proceso de filtrado, donde se validan ciertas cosas o eventos en detrimento de otros, y donde el proceso de exclusión es incluso más revelador que el de selección. Luego, "los mindscapes se institucionalizan en (dominios), grandes redes de prácticas interrelacionadas, ideas, artefactos, instituciones, etc. Estos dominios operan en medios diversos, tiene metas distintas y ocupan nichos diferentes en el tiempo y el espacio" (Ronström, 2005, p. 4). Como puede notarse, Ronström no vuelve sobre el objeto patrimonial mismo, sino sobre los procesos constituyentes en los que aparecen o desaparecen tales dispositivos, prácticas, instituciones y conceptos. De este modo, en cualquier lugar coexisten diversos mindscapes, anclados en la diversidad temporal e institucional, compitiendo o cooperando en diversos niveles y momentos.

Uno de los grandes argumentos con los cuales se "vendió" en la modernidad la práctica patrimonial es el de que ella era portadora y garante de la identidad colectiva. Evidentemente, tal aserto estaba ligado a la noción de una memoria esencial contenida en el patrimonio, la que aseguraría la conexión directa con el fondo ancestral, con la raíz identitaria. Sin embargo, como anota agudamente Kaufmann (2015, p. 24): "Todos la emplean como si remitiera a una evidencia, mientras que su definición incierta oculta apuestas políticas considerables". A renglón seguido, Kaufmann pasa revista a los errores y malos entendidos que el concepto de identidad, gran coartada del patrimonio, oculta: la identidad no está en ningunas raíces, sino "del lado de la sujetividad y de la producción de sentido" (Kaufmann, 2015. P. 26): esto es, en tanto no responde ya a una pregunta existencial, sino que "La identidad se deduce, antes que nada, de una subjetividad que actúa con vistas a producir un sentido que ya no está dado por el lugar social ocupado" (Kaufmann, 2015, p. 25). De ahí el que sea un concepto relativamente reciente, ligado a la modernidad en Occidente, a procesos biopolíticos de control poblacional, y que tuvo una serie de cambios a lo largo del tiempo, hasta llegar hoy a un estado donde se despliegan dos alternativas: "Nuestra época tiene

\footnotetext{
38 Prefiero no traducir el término, ya que "paisajes mentales", un acercamiento burdamente literal, connota otras cosas en español.
} 
dos rostros: el de las nuevas aperturas para el despliegue de subjetividades y el del auge de los fundamentalismos identitarios" (Kaufmann, 2015, p. 34). Los referentes a una u otra posición cambian estratégicamente su posición frente a un "patrimonio". Además, la erosión del estado-nación moderno, la emergencia de afiliaciones a varias nacionalidades simultáneas, la globalización económica con efectos sobre el trabajo, las migraciones y desplazamientos modifican este mapa identitario y producen o, bien, repliegues o despliegues identitarios.

$\mathrm{El}$ segundo error consiste en confundir los datos de trámite administrativo de los individuos, correspondientes a datos objetivos (edad, sexo, nivel educativo...), con la identidad. Así, tales datos fijos (la identificación) se confunden con el proceso siempre en movimiento de la identidad. Relacionado con este, un tercer error quiere dar a la identidad una fijeza, cuando este está en permanente proceso de reformulación. De nuevo, y como con el patrimonio, la identidad tiende a esencializarse y objetivarse, ignorando su matriz semiótica de autoproducción constante, su fecha histórica de constitución, las condiciones dentro de las cuales se adoptó y los avatares que lo alejan de tales coordenadas y piden su reformulación.

\section{Banalidad y volatilidad contemporánea}

El sesgo monumental, tan presente en las declaratorias de la UNESCO sobre bienes tanto culturales como naturales, se enfrenta con una tendencia que si bien nació con las propuestas artísticas de algunas vanguardias del siglo XX, se generalizó a la cultura y hoy ocupa un sitio aparentemente indestronable: la centralidad de lo banal ${ }^{39}$. Si el monumento (o el entorno idílico o el paisaje) convocaban figuras paradigmáticas que suponían valores trascendentes y ejemplares (recuérdese el carácter ejemplarizante y moral del patrimonio al seleccionar solo aquello que refrenda la bondad y el heroísmo, como resultado de las decisiones políticas patrimoniales), el lugar común, lo banal y lo cotidiano van a desplazarlo, reivindicando el tejido de lo corriente como matriz del gusto. Esta rebelión antiaristocrática trae al centro de la urbe contemporánea figuras de cómics elevadas a la categoría de monumentos, caricaturas de deidades o mitos; expande la noción de arte público al grafitti (antes mero vandalismo); lleva a la galería de arte los detritus; populariza el reality show o las conversaciones radiales sobre la intimidad llevadas al centro de la arena pública, la que, de paso, engulle ávida los últimos tweeter o actualizaciones en redes sociales de celebridades mayores y menores...

Este declive de la agenda moral patriarcal y burguesa inmersa en el patrimonio cívico y cultural parece, hoy día, irrecuperable, aunque plantea también otra moralidad alterna: nos recuerda los pies de barro de los poderosos,

39 Al respecto Jost, F. 2012, Danto, Arthur C., 2004, De Certeau, M., 1999. 
su pertenencia a la humanidad que los mira desde la galería esperando que se expongan sus errores y achaques, reivindica el derecho warholiano a los "quince minutos de fama" al que todos tendríamos derecho, al voyerismo como contrapartida del Big Brother electrónico, al hacer realidad el dictum de Beuys "todos somos artistas", a la sensiblería y el kistch y la emocionalidad derivada de la participación mediatizada en todos los momentos y eventos memorables o banales (desastres ${ }^{40}$, bodas y divorcios, muertes trágicas, confesiones en vivo y en directo, etc.). Frente al monumento, el contramonumento o la vandalización, la demolición de las obras e imágenes del tirano destronado, del referente nacional al local, de la glorificación de lo excepcional a la de lo corriente, del héroe histórico al antihéroe marginal o antisistema, de la utopía a las heterotopías. En un resumen muy apropiado del profesor Zalamea, el contexto en donde todo esto sucede se caracteriza por "la volatilidad contemporánea — su ligereza y plasticidad, su proclividad creativa y, al tiempo, su incapacidad de contrapesar y decantar, su ausencia de espiritualidad y sentimiento- y constituye ahora, ocho décadas después de Musil, nuestro pan de cada día." (Zalamea, 2004, p. 46).

\section{Turismo y patrimonio}

Una de las formas contemporáneas más poderosas de potenciar los patrimonios culturales y ponerlos al servicio de objetivos varios (sociales, económicos, etc.) es la de su ofrecimiento a la industria turística. A pesar de las quejas y prevenciones, el patrimonio es hoy uno de los objetivos más nítidos y buscados por los viajeros y agencias, al generalizarse la movilidad y al aparecer nuevos consumidores y demandas antes poco presentes en este mercado.

Las estrategias de city branding y de mercadeo de ciudades utilizan, quiérase o no, al patrimonio como otro de sus reclamos y carnadas, y la creación de oficinas gubernamentales, legislaciones locales y espacios de formación para servicios a la industria lo comprueban. Sin embargo, es evidente la distorsión y desfase entre los receptores de los beneficios (el sector privado en los países capitalistas), los efectos (urbanísticos, sociales, de cambios no deseados de uso de zonas, de expulsión de pobladores, parahotelería, etc.), y la posterior socialización de perjuicios y problemas, que supuestamente debe resolver el Estado a través de agencias locales, casi siempre desbordadas y poco eficientes.

En otro sentido, aunque complementario, la discusión sobre los usos del patrimonio cultural cobra aquí otra ruta: el turismo hace parte de esas nuevas fuerzas motrices que reconfiguran la cultura mundial y local, pero lo hace desde premisas simplistas, hegemónicas, y guiadas por intereses plenamente asép-

40 Zalamea habla de la "banalización del horror" (2004, p. 56), que hace eco a la denunciada "banalización del mal", acuñada por Hanna Arendt (1999). En otro registro y orilla ideológica, Mario Vargas Llosa (2012) lo hará acerca de la banalización de la cultura en "La civilización del espectáculo", un libro con reminiscencias debordianas, y una curiosa mezcla de nostalgia y moralización cultural. 
ticos: calidad de agua y alimentación, acceso a servicios que ni siquiera los nativos tienen, confort (o aventura, en el caso de un turismo "mochilero"), rarezas y exotismos a la mano, precios y presupuestos, seguridad.... Ello guía al diseño de "experiencias" patrimoniales completamente falseadas y pasteurizadas, objetivadas y pautadas más por el recreacionista que por la comunidad que se supone receptora del patrimonio o el experto que investiga su sentido y origen. Evidentemente, el patrimonio no solo habla del pasado, sino de los cambios, su estado presente y su proyección futura, pero el espectáculo turístico lo congela en el tiempo, en una ficción aceptable y políticamente correcta, a la medida de los usuarios, naturalizando narrativas y prácticas que son el resultado de negociaciones, pujas y dilemas incluso muy recientes ${ }^{41}$.

Smith (2006, p. 11) comienza el primer capítulo de su libro sobre los usos del patrimonio con la contundente afirmación de que "no existe tal cosa como el patrimonio", para concretar el que lo existente son no tanto "cosas" u "objetos" patrimoniales, como un conjunto de valores y significados, una práctica cultural involucrada en la construcción y regulación de un espectro de valores y de formas de entender. Tales prácticas son inherentemente políticas y discordantes", pero en ellas se refleja el presente y su forma de relacionarse con la herencia patrimonial Esta dimensión está, por supuesto y generalmente, por fuera de cualquier experiencia turística organizada. Al no ser esencialmente sobre objetos, monumentos, edificios, o cualquier materialidad, el patrimonio se referirá a los apegos, identificaciones y pertenencias, a lazos emocionales. Incluso, Smith (2006) también mostrará cómo la presencia de grupos culturales subalternos en los museos se ha de regir por la misma programación discursiva y museal hegemónica, al utilizar los mismos criterios patrimoniales aceptados en las exhibiciones donde se les permite entrar al sacrosanto recinto de la salvaguardia patrimonial.

Sin embargo, la reconfiguración del mapa patrimonial corre, en gran medida, por cuenta de la industria del turismo y de los caprichos de clientes y agencias. Las regulaciones gubernamentales, como se anotó antes, llegan post festum, pero estos organismos estatales no intervienen usualmente, a través de sus agencias o de convocatorias para indagación y creación y en la oferta a través de una alianza público-privada de experiencias patrimoniales mucho más densas simbólicamente, lo que evidentemente no quiere decir que sean aburridas,

41 Entrar en el "pacificado" espacio del Metro Cable en Medellín, sin que aparezcan las pugnas territoriales pasadas y presentes en las narrativas y guianzas. O, al contrario, resaltar en otra experiencia turística, las "tintas negras" sin reconocer cambios y mejoras. La apropiación del patrimonio aparece en experiencias muy diversas, validadas no solo desde un discurso patrimonial único y modélico y sus prácticas aparentemente asépticas, sino desde narrativas y prácticas heterogéneas: al respecto, no deja de ser irónico el que para construir el Museo Casa de la Memoria en Medellín, se haya demolido un espacio urbano lleno de vivencias e historias, pero cuya memoria desapareció con los pobladores dispersos en nombre del derecho y la necesidad del recuerdo de lo traumático. 
académicas o solemnes, pero sí que recreen el patrimonio en términos de sus relaciones de sentido y de apropiación y uso ${ }^{42}$.

\section{Propuestas y operaciones patrimoniales}

En la tesis de Maestría en Estudios Internacionales Museísticos, Von Unge (2008, p. 42) examina un problema práctico, el de la organización de las colecciones patrimoniales de un museo sueco. Ello la lleva a mostrar cómo existe una evidente reconceptualización categorial y de estrategias para desplegar el patrimonio. En resumen, muestra cómo la actividad pasa de ser orientada de forma general a serlo particularmente, del énfasis material al contextual, de la sistematización a la inspiración, y de documentar el presente para el futuro, a documentar el presente para hoy y mañana.

Este conjunto de procesos y reglas simples permite pensar, inicialmente, en que las curadurías o encargos comisariales, tan frecuentes en las artes, podrían ser convocadas para gestionar trabajo con el patrimonio, de modo que un individuo o colectivo presentaran su versión, más inspirada que sistemática y "científica", desplegando la posibilidad de discusiones y de nuevas formas de abordar, exhibir y relacionarse con tales herencias culturales.

La desustancialización del patrimonio, es decir, el paso del patrimonio como objeto al patrimonio como relación, abre la puerta a pensar en un patrimonio inherentemente relacional, polémico, sin valores permanentes, un campo de discusión móvil y cambiante que interpela y dice más del presente que del pasado. Del mismo modo, la recuperación de contextos, desenfatizando también la materialidad de los objetos mismos, una práctica museal ya extendida, permite operar sobre los patrimonios de un modo creativo y refrescante, sin aislar a estos de la trama donde operaron u operan hoy. Al tiempo, la actividad de documentar recobra un pie en el presente y no solo en el futuro, permitiendo intervenciones de los interpelados por los patrimonios con los que se les relaciona.

El determinar el uso del patrimonio cultural es el elemento capital de esta agenda: debe revisarse la retórica de la "identidad", del legado cultural idealizado per se, y abordar las formas complejas en las que Estado y agencias privadas concurren en este campo. No se trata solo de legislar o de acudir a remediar situaciones provocadas por el expolio privado, sino de definir cómo y dónde interviene el Estado, por sí mismo, y dónde acude al recurso de asociarse con entidades privadas y comunidades, y establecer claramente el para qué de estas acciones, partiendo del sentido que el patrimonio cultural tiene para los colectivos que han de apropiárselo. Del mismo modo, la tecnología es un

42 Particularmente ilustrativo es el trabajo de Staiff, R., Bushell, R. and Watson S. (eds.) (2013), donde exploran diversos espacios nacionales de turismo patrimonial las formas de representación, encuentro, compromiso, actuación y falsificación de la memoria cultural. 
poderoso aliado para la identificación, gestión y divulgación patrimonial, pero a condición de tener claros sus límites y posibilidades en términos de los usos y apropiaciones que del patrimonio puedan hacerse a través de ella.

La gestión patrimonial y las políticas del sector no son hoy de puro monopolio estatal: de hecho, decisiones tomadas en otros ámbitos (educación, transporte, medio ambiente, salud...), ya sea desde el Estado o por parte otros agentes, tienen efectos en la trama del patrimonio y sus alcances. Esta gestión debe estar atenta a esas formas cambiantes de relación donde surge lo patrimonial ${ }^{43}$, ofreciendo modos creativos y participativos de co-crear situaciones y experiencias patrimoniales que echan mano de muchas estrategias de activación y visibilización ${ }^{44}$, alejadas de rutinas repetitivas y validadoras de un modelo patrimonial ya en cuestión por su irrelevancia en las vidas de los habitantes de un territorio, a quienes deberían interpelar y propiciar la labor de seguir construyendo sentidos, la verdadera labor de los herederos patrimoniales.

\section{Conclusiones}

Para redondear de algún modo este mosaico de temas, tal vez nada mejor que acudir aquí a un concepto foucaultiano: al definir un dispositivo, Foucault (en una entrevista concedida en 1984) explicaba:

Lo que trato de indicar con este nombre es, en primer lugar, un conjunto resueltamente heterogéneo que incluye discursos, instituciones, instalaciones arquitectónicas, decisiones reglamentarias, leyes, medidas administrativas, enunciados científicos, proposiciones filosóficas, morales, filantrópicas, brevemente, lo dicho y también lo nodicho, éstos son los elementos del dispositivo. El dispositivo mismo es la red que se establece entre estos elementos. ${ }^{45}$

Esta noción es plenamente válida y fructífera para examinar el dispositivo patrimonial, identificando los discursos (uno de carácter oficial, hegemónico, de agencias gubernamentales y de algunos académicos, mientras que, como he tratado de insinuar, van emergiendo otros de carácter crítico al lado de otros más de afinidad tecnocrática), las instituciones (desde UNESCO o ICOM ${ }^{46} \mathrm{en} \mathrm{el}$ plano internacional, a las diversas instancias nacionales y locales, ministerios, institutos y secretarías de cultura, etc., y numerosas agencias privadas), las decisiones, leyes y medidas administrativas (emanados de las instituciones,

43 El atentado donde resultó dañada una escultura de Fernando Botero en 1995, y la posterior instalación de una escultura gemela al lado de la ruina en el Parque San Antonio en Medellín, reflejan claramente una atención gubernamental oportuna a un lugar y un objeto que se convierten en memoria. El caso contrario puede ser ilustrado de infinitas maneras.

44 Con ejemplos como los columbarios intervenidos en el Cementerio Centra de Bogotá por Beatriz González en 2009, la realizada en el Palacio de Justicia en Bogotá por Doris Salcedo en 2007, la realizada a 5 esculturas en Medellín en 2015 con el lenguaje Steampunk.

45 Citado por Agamben, G., 2005. La entrevista está consignada en Foucault (1985).

46 Siglas en inglés del Instituto internacional de museos. 
con motivaciones y objetivos bien diversos), los enunciados científicos ( definiciones de patrimonio, de cultura, de identidad, de memoria, venidas de varios ámbitos disciplinares...), las proposiciones filosóficas y morales (el derecho a la memoria, los derechos culturales, el carácter civilizatorio del arte, la ciudadanía cultural, los usos del patrimonio...), etc. Los desajustes entre estos elementos de la red, sus desfases y exigencias, la adopción de tecnologías de gestión, etc., van dibujando el panorama actual de la relación con el patrimonio cultural en diversos espacios, y las pugnas y estrategias para ignorar o reconceptualizar los avatares a los que este dispositivo está hoy sometido.

El patrimonio es ahora un campo dinámico de discusión, ya no territorio exclusivo de especialistas, donde aristas como las del turismo, las nuevas identidades sociales, los imaginarios de nacionalidad, las ideas sobre la memoria y el pasado, las tecnologías, las industrias culturales, las redefiniciones de lo cultural, etc., rearman el dispositivo patrimonial de muchas formas, no solo desde la dimensión universalizante como la proclamada por UNESCO, ni la idea del monopolio de la memoria social subsumida por la patrimonialización. Es por ello que, como quiso argumentarse en este artículo, hay un gran potencial de indagación y de apertura de nuevas maneras de abordar, conceptualizar y proponer otras relaciones con el patrimonio, tarea que implica creatividad, trabajo multidisciplinario, convocatoria a la ciudadanía y a los hacedores de políticas públicas. Cualquier elemento faltante en esta ecuación seguirá provocando desfases y deformaciones, impidiendo que los potenciales sociales y culturales de este universo, logren convocar nuevas formas de estar juntos.

\section{Referencias bibliográficas}

Agamben, Giorgio (2005, octubre 12). Qué es un dispositivo. Conferencia. Universidad Nacional de la Plata, Argentina. En línea en http://www.trelew.gov.ar/web/files/LEF/SEM03-AgambenQueesunDispositivo.pdf

Allier Montaño, Eugenia (2008). Los Lieux de mémoire: una propuesta historiográfica para el análisis de la memoria. Historia y Grafía, n. ${ }^{\circ} 31$, pp. 165-192.

Álvarez Cano, María (2015). Derecho al olvido en internet: el nuevo paradigma de la privacidad en la era digital. Madrid: Reu- Universidad CEU-San Pablo.

Arendt, Hanna (1999). Eichmann en Jerusalem. Un informe sobre la banalidad del mal. 2. ${ }^{a}$ ed. Barcelona: Lumen.

Atkinson, Jeanette (2014). Education, Values and Ethics in International Heritage: Learning to Respect. Farnham (England): Ashgate Publishing.

Avilés Flórez, Pablo (2012). El patrimonio cultural: función social y relaciones interdisciplinarias. MOUSEION, n. ${ }^{\circ}$ 13, set-dez, pp 76-96.

Bendix, Regina F., Egert, Aditya, Peselmann, Arnika (eds.). (2012). Heritage Regimes and the State. Göttingen: Universitätsverlag Göttingen. Göttingen Studies in Cultural Property, Volume 6. 
Choay, Françoise (2001). The Invention of the Historic Monument. Cambridge: Cambridge University Press.

Danto, Arthur C. (2004). La transfiguración del lugar común. Una filosofía del arte. Buenos Aires: Paidós. Debray, Régis (2001). Introducción a la mediología. Barcelona: Paidós.

De Certeau, Michel (1999). La invención de lo cotidiano. México: Universidad Iberoamericana/ITESO/ Centro Francés de Estudios Mexicanos y Centroamericanos.

Déotte, Jean-Louis (2013). La época de los aparatos. Buenos Aires: Adriana Hidalgo.

Dicks, Bella (2004). Culture on Display: The Production of Contemporary Visitability. Maidenhead, England: Open University Press- McGraw Hill.

Foucault, Michel (1985). Saber y verdad. Madrid: La Piqueta

Gamboni, Dario (2014). La destrucción del arte. Iconoclasia y vandalismo desde la Revolución Francesa. Madrid: Cátedra.

González Alcantud, José A. (2012). El malestar en la cultura patrimonial. La otra memoria global. Barcelona: Anthropos.

Huyssen, Andreas (2002). En busca del futuro perdido. Cultura y memoria en tiempos de globalización. México: Fondo de Cultura Económica.

Innocenti, Perla (ed.) (2014). Migrating Heritage: Experiences of Cultural Networks and Cultural Dialogue in Europe. Farnham (England): Ashgate Publishing.

Jost, François (2012). El culto de lo banal. De Duchamp a los reality shows. Buenos Aires: Libraria.

Kaufmann, Jean-Claude (2015). Identidades. Una bomba de relojería. Barcelona: Ariel.

Lowenthal, David (1998). The Heritage Crusade and the Spoils of History. Cambridge: Cambridge University Press.

Maier, Charles (1988). The Unmasterable Past: History, Holocaust, and German National Identity. Cambridge (Mass.) and Londres: Harvard University Press.

Matarasso, François and Landry, Charles (1999). Balancing Act: Twenty-One Strategic Dilemas in Cultural Policy. Strasbourg: Council of Europe.

Mayos, Gonçal (s.f.). Modernidad y racionalidad razón geométrica versus razón dialéctica. Disponible en https://www.academia.edu/4390009/Modernidad _ y _ racionalidad. Raz\%C3\%B3n_geom\%C3\%A9trica _ versus _raz\%C3\%B3n _dial\%C3\%A9ctica

Nora, Pierre (ed.) (2001). Le Lieux de Mémorie. 2. a ed., París: Gallimard, (3 tomos)

Richmond, Alison and Bracker, Alison (eds.) (2009). Conservation: Principles, Dilemmas and Uncomfortable Truths. London: Butterworth-Heinemann and The Victoria and Albert Museum,

Robin, Régine. (2003). La memoria saturada. Buenos Aires: Waldhuter.

Ronström, Owe. (2005). Memories, tradition, heritage. In Ronström, Owe \& Palmenfelt, Ulf (eds.). Memories and Visions. Studies in Folk Culture IV. Departement of Estonian and Comparative Folklore \& Department of Ethnology, University of Tartu. Disponible en https:// www.diva-portal.org/smash/get/diva2:304792/FULLTEXT01.pdf 
Rusu, Mihai Stelian. (2015). From the Will to Memory to the Right to Be Forgotten A Paradigm Shift in the Culture of Remembering. Philobiblon. Transylvanian Journal of Multidisciplinary Research in Humanities, V. XX, n. ${ }^{\circ}$ 2, pp. 384-411.

Salazar, Juan Francisco and Bushell, Robyn (2014). Heritage for sale: Indigenous tourism and misrepresentations of voice in northern Chile. In Bushell and Watson (eds.), Staiff, 187 ss.

Smith, Laurajane. (2006). The Uses of Heritage. London and New York: Routledge.

Smith, Laurajane (2011). El ıespejo patrimonialı. ¿Ilusión narcisista o reflexiones múltiples? Antipod. Rev. Antropol. Arqueol, n. ${ }^{\circ}$ 12, Bogotá (Enero-Junio), pp. 39-63.

Staiff, Russell, Bushell, Roby and Watson, Steve (eds.) (2014). Re-imagining Heritage Interpretation: Enchanting the Past-Future. Farnham (England): Ashgate Publishing.

Staiff, Russell, Bushell, Roby and Watson, Steve (eds.) (2013) Heritage and Tourism: Place, Encounter, Engagement. New York: Routledge.

Stiegler, Bernard (1998). Leroi-Gourhan: linorganique organize. Cahiers de médiologie $N^{\circ} 6$. Pourquoi des médiologues, pp. 187-194.

Thuen, Trond (2004). Culture as Property? Some Saami Dilemmas. In Kasten, Erich (ed.). Properties of Culture - Culture as Property. Pathways to Reform in Post-Soviet Siberia. Berlin: Dietrich Reimer Verlag, pp. 87-108.

Vargas Llosa, Mario (2012). La civilización del espectáculo. Madrid: Alfaguara.

Von Unge, Elin Nystrand (2008). When Culture Becomes Heritage. In search of the Samdok discourse of collecting contemporary heritage. Masterss Thesis. International Museum Studies. Götemborgs Universitet.

Weinrich, Harald (1999). Leteo. Arte y crítica del olvido. Madrid: Siruela.

Wilson, Edward O. (1998). Consilience. New York: Alfred A. Knopf.

Zalamea Traba, Fernando (2004). Ariadna y Penélope. Redes y mixturas en el mundo contemporáneo. Oviedo: Nobel (Premio Internacional de Ensayo Jovellanos, 2004). 